\title{
Constructing a Galactic coordinate system based on near-infrared and radio catalogs
}

\author{
J.-C. $\mathrm{Liu}^{1,2}$, Z. Zhu ${ }^{1,2}$, and B. $\mathrm{Hu}^{3,4}$ \\ 1 Department of astronomy, Nanjing University, Nanjing 210093, PR China \\ e-mail: [jcliu;zhuzi]@nju.edu.cn \\ 2 key Laboratory of Modern Astronomy and Astrophysics (Nanjing University), Ministry of Education, Nanjing 210093, PR China \\ 3 Purple Mountain Observatory, Chinese Academy of Sciences, Nanjing 210008, PR China \\ 4 Graduate School of Chinese Academy of Sciences, Beijing 100049, PR China \\ e-mail: hubo@pmo.ac.cn
}

Received 24 March 2011 / Accepted 13 October 2011

\begin{abstract}
Context. The definition of the Galactic coordinate system was announced by the IAU Sub-Commission 33b on behalf of the IAU in 1958. An unrigorous transformation was adopted by the HIPPARcos group to transform the Galactic coordinate system from the FK4-based B1950.0 system to the FK5-based J2000.0 system or to the International Celestial Reference System (ICRS). For more than 50 years, the definition of the Galactic coordinate system has remained unchanged from this IAU1958 version. On the basis of deep and all-sky catalogs, the position of the Galactic plane can be revised and updated definitions of the Galactic coordinate systems can be proposed.

Aims. We re-determine the position of the Galactic plane based on modern large catalogs, such as the Two-Micron All-Sky Survey (2MASS) and the SPECFIND v2.0. This paper also aims to propose a possible definition of the optimal Galactic coordinate system by adopting the ICRS position of the Sgr A* at the Galactic center.

Methods. The near-infrared 2MASS point source catalog and the SPECFIND v2.0 catalog of radio continuum spectra are used to determine the mean position of the Galactic plane on the celestial sphere. By fitting the data to an ideal Galactic equator, the parameters defining the Galactic coordinate system are obtained.

Results. We find that the obliquity of the Galactic equator on the ICRS principal plane is about $0.4^{\circ}$ (2MASS) and $0.6^{\circ}$ (SPECFIND v2.0) larger than the $\mathrm{J} 2000.0$ value, which is widely used in coordinate transformations between the equatorial $(\alpha, \delta)$ and the Galactic $(\ell, b)$. Depending on the adopted parameters, data, and methods, the largest difference between the resulting Galactic coordinate systems is several arcminutes. We derive revised transformation matrices and parameters describing the orientation of the Galactic coordinate systems in the ICRS at the 1 milliarcsecond level to match the precision of modern observations.

Conclusions. For practical applications, we propose that a revised definition of the Galactic coordinate system should be required in the near future.
\end{abstract}

Key words. astrometry - reference systems - Galaxy: general - catalogs

\section{Introduction}

The Galactic coordinate system (GalCS) is important for studies of the Galactic structure, kinematics, and dynamics. In the GalCS, the principal plane (or the Galactic equator) coincides with the plane of the Galaxy and the Galactic longitude of $0^{\circ}$ is defined as the direction pointing to the Galactic center (GC). According to the above criteria, the IAU sub-commission 33b summarized previous observations and defined the GalCS in the framework of the B1950.0 FK4-based reference system in 1958 (Blaauw et al. 1960). The standard GalCS subsequently adopted was that for which the pole was based primarily on the distribution of neutral hydrogen in the inner parts of the Galactic disk. Murray (1989) discussed the transformation of the GalCS from the FK4 to the FK5 system and derived the orientation of the GalCS at the epoch of J2000.0, and his method was adopted by the HiPPARcos team as a standard transformation.

Liu et al. (2011) discussed the history of the GalCS, found that a J2000.0 GalCS, which was transformed from the original IAU1958 definition using unrigorous methods does not match the physical features of the GalCS, and pointed out that there may then be some confusion, when applying the Galactic coordinates. Here we list the transformation matrix and the parameters for the sake of comparison. The transformation matrix $(3 \times 3)$ from the equatorial to the Galactic coordinate system can be written as

$\mathcal{N}=\mathcal{R}_{3}\left(90^{\circ}-\theta\right) \mathcal{R}_{1}\left(90^{\circ}-\delta^{\mathrm{p}}\right) \mathcal{R}_{3}\left(90^{\circ}+\alpha^{\mathrm{p}}\right)$,

where $\left(\alpha^{\mathrm{p}}, \delta^{\mathrm{p}}\right)$ is the equatorial coordinates of the north Galactic pole (NGP) and $\theta$ is the position angle of the Galactic center (GC) at the NGP with respect to the equatorial pole. These three parameters were defined at the epoch of B1950.0 by the IAU in 1958 (Blaauw et al. 1960). Their numerical values, referred to the FK5-based J2000.0 reference system, were derived as

$$
\begin{aligned}
\alpha_{\mathrm{J} 2000.0}^{\mathrm{p}} & =12^{\mathrm{h}} 51^{\mathrm{m}} 26^{\mathrm{s}} .2755, \\
\delta_{\mathrm{J} 2000.0}^{\mathrm{p}} & =+27^{\circ} 07^{\prime} 41^{\prime \prime} \cdot 704, \\
\theta_{\mathrm{J} 2000.0} & =122^{\circ} .93191857, \\
i_{\mathrm{N}} & =90^{\circ}-\delta_{\mathrm{J} 2000.0}^{\mathrm{p}}=62^{\circ} .8717,
\end{aligned}
$$

where $i_{\mathrm{N}}$ is the inclination of the Galactic plane. 
Starting from the J2000.0 system, Liu et al. (2011) derived the GalCS in the ICRS by using the bias matrix $\mathcal{B}$, and the authors also tentatively proposed a new definition of the GalCS using the position of the compact radio source Sagittarius A* (Sgr A*). They only used a single observation of the GC (ICRS position of Sgr $A^{*}$ ) to construct a new coordinate system, failing to using other data to ensure that this revised definition is the optimal choice. Large astrometric and photometric catalogs such as the Two-Micron All-Sky Survey (2MASS) and other catalogs at radio wavelength such as SPECFIND v2.0 have provided us with deeper and more homogeneous observational data to estimate more reliably the position of the Galactic plane. To construct an optimal GalCS, they also ensured that there was an accurate match with the real Galactic plane on the celestial sphere, namely, that the principal plane $(x-y$ plane) corresponds to the median plane of the Milky Way and its $x$-axis pointing to the GC.

It is known that the Milky Way is a disk-like Galaxy in which our Sun is located. The scale height of the thick disk is about $1350 \mathrm{pc}$ (Gilmore \& Reid 1983) or 1048 pc (Veltz et al. 2008). Since the vertical displacement of the Sun above the Galactic disk ( $\sim 15$ pc) (Zhu 2009) is much smaller than the Galactocentric distance of the Sun $(\sim 8.0 \mathrm{kpc})$ (Reid 1993) and the scale height of the Galaxy, we can assume that the Sun is in the middle of the Galactic disk so that the Milky Way is an "edge-on" galaxy seen from the Sun, which leads to equal numbers of member stars above and below the mean Galactic plane. A deep and homogeneous all-sky survey should reveal the edgeon structure of the Milky Way and the stars should be evenly distributed above and below a median great circle on the celestial sphere, which is the optimal Galactic plane we intend to find. In this paper, we select and analyze data from the latest near-infrared and radio catalogs to calculate the orientation of the optimal GalCS in the ICRS.

In Sect. 2, we describe the properties of the data that we use to fit the mean position of Galactic plane. Section 3 presents the results for the Galactic plane and the GC positions, and we discuss our revised definitions of the GalCS in Sect. 4. Finally, in Sect. 5 we discuss and summarize our main results and draw some conclusions.

\section{Data}

We attempt to estimate the position of the Galactic plane using data describing the all-sky distribution of stars. Our goal is to calculate three parameters $\left(\alpha^{\mathrm{p}}, \delta^{\mathrm{p}}, \theta\right)$ that describe the orientation of the GalCS in the ICRS. A deep all-sky catalog with stars complete to a certain magnitude would reproduce a smooth Galactic belt on the celestial sphere and a highest star density in the direction of the GC if we did not consider interstellar extinction and the vertical distance of the Sun above the Galactic disk.

A catalog has to meet several criteria if it can be used to determine reliably the position of the Galactic plane. First, it must cover the area of the whole disk at low Galactic latitudes. Secondly, it must be affected by little or no interstellar extinction so that the two dimensional (on the celestial sphere) distribution of the stars is uniform. Infrared or radio catalogs usually meet this criteria but optical catalogs are strongly affected especially at low Galactic latitudes. Thirdly, it should contain a sufficient number of stars in the framework of the ICRS. The positional precision of the individual stars are not crucial because we rely on a statistical least squares fit method (see the next section) to obtain the most reliable estimate of the mean position of the Galactic plane. Proper motions of stars are not needed either,

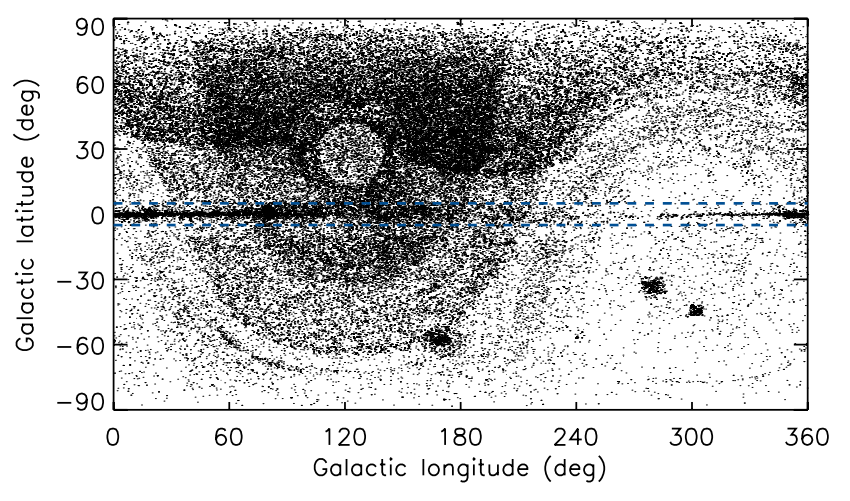

Fig. 1. The sky distribution of the SPECFIND v2.0 catalog in the $\mathrm{J} 2000.0 \mathrm{GalCS}$. The dashed lines are horizontal at $b=-5^{\circ}$ and $b=+5^{\circ}$

because the newly defined GalCS is designed to be epoch independent and fixed with respect to the ICRS.

We choose the 2MASS point source catalog (Cutri et al. 2003; Skrutskie et al. 2006) at near infrared wavelengths and the SPECFIND v2.0 (Vollmer et al. 2010) catalog in the radio band which satisfy all of the above criteria, in our analysis here.

\section{1. $2 M A S S$}

The 2MASS catalog was constructed from uniform observations of the entire sky (covering $99.998 \%$ of the celestial sphere) in three near-infrared bands: $J(1.25 \mu \mathrm{m}), H(1.65 \mu \mathrm{m})$, and $K_{\mathrm{s}}(2.16 \mu \mathrm{m})$. The limiting flux in each band is about $1 \mathrm{mJy}$. The 2MASS contains positions and photometric parameters of 470992970 sources, but no proper motions. The positions of stars are calibrated to the Tycho- 2 catalog and the astrometric accuracy is 70-80 mas in the magnitude range of $9<K_{\mathrm{s}}<14$ mag. Figure 13 of Skrutskie et al. (2006) shows a beautiful pictures of our Galaxy across the Sky. The 2MASS scans have a typical signal-to-noise ratio of ten and is $>99 \%$ complete above 15.8 , 15.1 , and $14.3 \mathrm{mag}$ for $J, H$, and $K_{\mathrm{s}}$, respectively. We choose a complete set of data to fit the Galactic equator by limiting the data we consider to those for which $11<J<15.8$.

\subsection{SPECFIND v2.0}

The new release of the SPECFIND radio cross-identification catalog (Vollmer et al. 2010) contains 3.76 million cross-identified radio objects, which has about $60 \%$ more sources than its first release in 2005. The positions of the radio objects in the SPECFIND v2.0 catalog were derived from 97 catalogs. Figure 1 depicts the projected distribution of objects in the J2000.0 Galactic coordinates system from which we can see clearly the Galactic plane, even though the whole catalog is not very homogeneous. In this sense, only objects at low Galactic latitudes are selected.

\section{The position of the Galactic plane in the ICRS}

\subsection{Fitting the position of the Galactic plane on the celestial sphere}

The orientation of the Galactic equator can be defined by the equatorial coordinates of the $\operatorname{NGP}\left(\alpha^{\mathrm{p}}, \delta^{\mathrm{p}}\right)$ or equivalently $\left(\alpha_{\mathrm{N}}, i_{\mathrm{N}}\right)$, where $\alpha_{\mathrm{N}}$ is the right ascension of the ascending node 


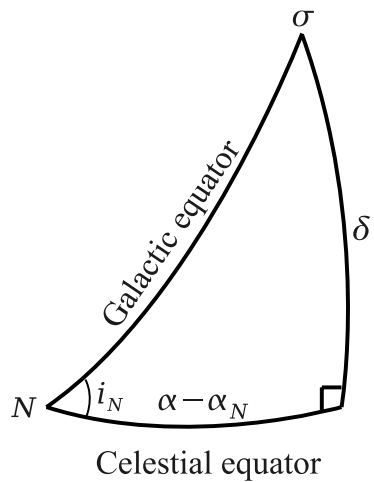

Fig. 2. The pattern of the Galactic plane and the celestial equator: $\sigma(\alpha, \delta)$ is a star on the Galactic plane. $N$ is the ascending node of the Galactic plane with respect to the celestial equator.

of the Galactic equator on the celestial equator and $i_{\mathrm{N}}$ is the inclination of the Galactic plane. They are related by

$\alpha_{\mathrm{N}}-\alpha^{\mathrm{p}}=90^{\circ}, i_{\mathrm{N}}+\delta^{\mathrm{p}}=90^{\circ}$.

Figure 2 shows the relationship between the Galactic plane and the equator. The equatorial coordinates $(\alpha, \delta)$ of a star $\sigma$ on the Galactic plane satisfies the equation

$\tan \delta=\sin \left(\alpha-\alpha_{\mathrm{N}}\right) \tan i_{\mathrm{N}}$,

in which $\alpha_{\mathrm{N}}$ and $i_{\mathrm{N}}$ are two parameters to be fitted from the catalog data.

In this paper, we ignore the weak interstellar extinction at the infrared and radio bands (which means that the survey observation is homogeneous) so that the Galaxy has a symmetrical structure as seen from the Sun. The optimal Galactic equator should be a great circle on the celestial sphere that is the closest fit to the density distribution pattern of the above catalogs: the position of the Galactic plane is where the highest star density appears in the direction of the Galactic longitudes, because stars should be concentrated on the real Galactic plane due to the gravitational attraction and rotation of the Milky Way. In other words, the great circle chosen should have an equal number of stars on both sides.

For the 2MASS point source catalog, we select stars within the magnitude range $11<J<15.8$. The upper limit to the magnitude range is the $99 \%$ completeness magnitude in the $J$ band as mentioned in Sect. 2.1, and the lower limit rejects bright stars close to the Sun. Owing to the local structure of the of the Galaxy, such as the Gould belt (Westin 1985) and the Galactic warp (Miyamoto et al. 1988), which may affect our results, we exclude nearby stars by applying a bright upper limit to our selected sample. The complete sample comprises about 56.7 percentage of stars in the whole 2 MASS catalog. The density map (360 bins in $\ell \times 180$ bins in $b$ ) of the selected stars in the J2000.0 Galactic coordinate system is shown in Fig. 3, which depicts a beautiful edge-on Galactic plane on the sky. The star density at high Galactic latitudes is much lower than closer to the Galactic plane and the influence of halo stars is not high.

Instead of fitting Eq. (3) to individual star coordinates $(\alpha, \delta)$, we choose to use the equatorial positions of the geometrical centers of each $1^{\circ}$-Galactic-longitude bin (360 in total), in order to derive a more accurate estimate of $\alpha_{\mathrm{N}}$ and $i_{\mathrm{N}}$. We analyze the 2MASS data in this way for the following reasons. The stellar density close to the GC is extremely high (which means that the weight of the data in the GC region is much larger than that

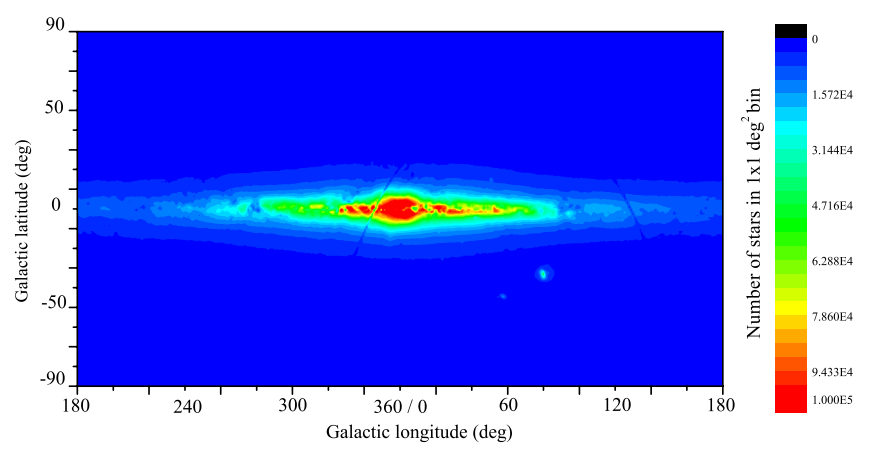

Fig. 3. The star density distribution of the 2 MASS point source catalog in the magnitude range $11<J<15.8$. The direction of GC $(\ell=0, b=$ 0 ) is plotted at the center.

in any other area), such that the small-scale structures close to the GC will strongly affect the resulting position of the Galactic plane. Our processing method assigns to data at all Galactic longitudes the same weight to avoid any biasing. The densities within the LMC and SMC areas are excessively high in the current study and are replaced with surrounding densities in subsequent calculations.

We first calculate the central Galactic coordinates of the 360 columns of the $360 \times 180$ density-distribution matrix and then transform $\left(\bar{\ell}_{k}, \bar{b}_{k}\right), k=1 \ldots 360$ into an equatorial coordinate system to derive the equatorial coordinates $\left(\bar{\alpha}_{k}, \bar{\delta}_{k}\right), k=1 \ldots 360$, by applying the previous $\mathbf{J} 2000.0$ transformation matrix in Eq. (1). In the next step, these equatorial coordinates are fitted with Eq. (3) to obtain $\alpha_{\mathrm{N}}$ and $i_{\mathrm{N}}$ for the first time. With the additional position angle $\theta_{\mathrm{J} 2000.0}$ in Eq. (2), we can write the new transformation matrix $\mathcal{N}$, according to which the updated Galactic coordinates of the stars and the updated density matrix of our samples can be obtained. Repeating the above processes, we derive converged parameters $\alpha_{\mathrm{N}}$ and $i_{\mathrm{N}}$, which have the numerical values

$\alpha_{\mathrm{N}}=282^{\circ} .987 \pm 0.032, i_{\mathrm{N}}=63^{\circ} .280 \pm 0.019$.

These values of $\left(\alpha_{\mathrm{N}}, i_{\mathrm{N}}\right)$ are equivalent to the IAU definition parameters $\left(\alpha^{\mathrm{p}}, \delta^{\mathrm{p}}\right)$ and we can quote them to infinite accuracy (here and hereafter to 1 milliarcsecond), if they were adopted as a definition

$\begin{aligned} \alpha_{2 \text { MASS }}^{\mathrm{p}} & =12^{\mathrm{h}} 51^{\mathrm{m}} 56^{\mathrm{s}} .7726, \\ \delta_{2 \mathrm{MASS}}^{\mathrm{p}} & =+26^{\circ} 43^{\prime} 11^{\prime \prime} .096 .\end{aligned}$

It should be understood that this is a numerical convention and does not necessarily imply that any given parameters and elements of the matrices (in the following equations) are known to the quoted accuracy. The standard $1-\sigma$ errors in above results reflect only the uncertainty in the data fitting and have no deep physical meanings.

Figure 4 illustrates the positions of the 360 bin centers, in which the gray curve shows the original value, whereas the black one is plotted after $\alpha_{\mathrm{N}}$ and $i_{\mathrm{N}}$ converge. The raw and reduced curve are almost evenly situated above and below $b=0$. They have similar shapes along the great circle $b=0$ and correspond to the old and new transformation matrix $\mathcal{N}$. The difference between the two curves reflects the small-angle orientation bias between the original and the final GalCSs.

In the SPECFIND v2.0 catalog (see Fig. 1), objects are not evenly distributed on the sky: the southern hemisphere was observed less than the northern. The global structure of this distribution has a strong effect on our results, even though the Galactic 


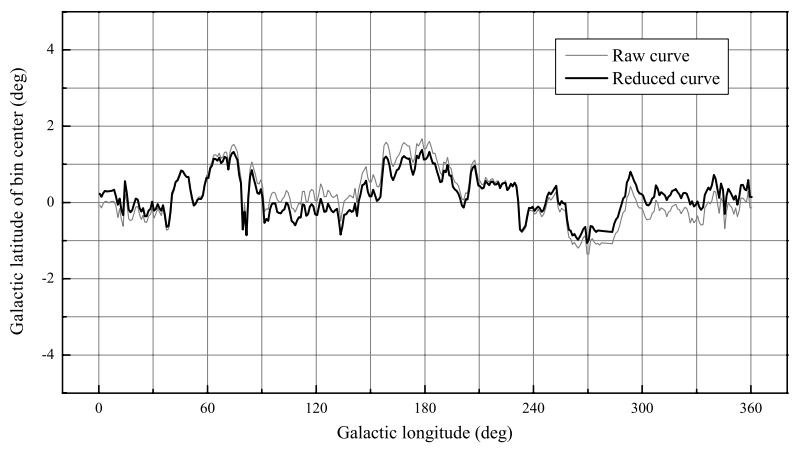

Fig. 4. The curve of the center in $1^{\circ}$ longitude bins. The gray curve is the original curve transformed by J2000.0 rotation matrix and the black one the reduced curve transformed by new matrix that is derived from 2MASS fitting.

structure can be clearly seen in Fig. 1 . We only retain the data at low Galactic latitudes $|b|<5^{\circ}$, which avoids possible biasing. We divide the belt $\left(0^{\circ} \leq l<360^{\circ},-5^{\circ}<b<5^{\circ}\right)$ into 360 bins, each one with $1^{\circ}$ (in $\left.\ell\right) \times 10^{\circ}$ (in $b$ ) coverage. The mean positions of objects in each bin $\left(\bar{\ell}_{\mathrm{k}}, \bar{b}_{\mathrm{k}}\right), k=1, \ldots, 360$ are then calculated and transformed into the $\mathbf{J} 2000.0$ equatorial coordinates $\left(\bar{\alpha}_{\mathrm{k}}, \bar{\delta}_{\mathrm{k}}\right)$, $k=1, \ldots, 360$. Similarly as we process the 2 MASS catalog, these 360 equatorial coordinates are used to to fit $\alpha_{\mathrm{N}}$ and $i_{\mathrm{N}}$ in Eq. (3) using a least squares method. After we obtain the resulting $\alpha_{\mathrm{N}}$ and $i_{\mathrm{N}}$, the equatorial to Galactic transformation matrix $\mathcal{N}$ can be derived by applying $\theta_{\mathrm{J} 2000.0}$. The equatorial coordinates of the raw data can then be transformed into the Galactic coordinates using the new matrix. In the next step, we recalculate the updated coordinates of the mean positions $\left(\bar{\ell}_{\mathrm{k}}, \bar{b}_{\mathrm{k}}\right)$ and fit Eq. (3) to get another pair of $\alpha_{\mathrm{N}}$ and $i_{\mathrm{N}}$ values. These procedures are repeated until the two objective parameters converge to the final values. The resulting parameters are

$\alpha_{\mathrm{N}}=282^{\circ} .699 \pm 0.031, \quad i_{\mathrm{N}}=63^{\circ} .513 \pm 0.020$,

or the coordinates of the NGP are

$$
\alpha_{\text {SPECFIND }}^{\mathrm{p}}=12^{\mathrm{h}} 50^{\mathrm{m}} 47^{\mathrm{s}} .6503
$$$$
\delta_{\text {SPECFIND }}^{\mathrm{p}}=+26^{\circ} 29^{\prime} 12^{\prime \prime} .412 \text {. }
$$

We note that the position angle $\theta_{\mathrm{J} 2000.0}$ (which indicates the position of the GC on the Galactic plane) is unaltered in iterative steps because we are only concerned with the position of the Galactic plane in both the 2MASS and SPECFIND v2.0 data sets. From Eqs. (4) and (5), we find that the fitted values of inclination $i_{\mathrm{N}}$ from the 2MASS and the SPECFIND v2.0 are about $0.41^{\circ}$ and $0.64^{\circ}$ respectively, larger than the $\mathrm{J} 2000.0$ reference values in Eq. (2). The ascending node fitted using the 2MASS data is about 7 arcsec eastward of the J2000.0 node, but the corresponding result using the SPECFIND v2.0 data is about 10 arcsec westward of the J2000.0 node. We emphasize here that our results depend on the choice of data (range of magnitude, type of stars, bandwidths, etc.), although both 2MASS and SPECFIND v2.0 are appropriate candidate catalogs to fit $\alpha_{\mathrm{N}}$ and $i_{\mathrm{N}}$ because of their advantages described above. The generated results in Eqs. (4) and (5) present, to a certain extent, the position of the Galactic plane in the near-infrared and radio continuum data sets based on modern observations. We confirm that complete and deeper long-wavelength surveys will provide more reliable estimates. Although there are many extremely large optical catalogs, such as UCAC3 (Zacharias et al. 2010),
USNO B1.0 (Monet et al. 2003), and the latest PPMXL catalog (Roeser et al. 2010) (of nearly one billion stars), they cannot be used in this study, mainly because of the non-isotropic interstellar extinction along different lines of sight, which destroys the homogeneity of the density distribution of stars in these optical catalogs.

\subsection{The position of the Galactic center in the ICRS}

The IAU working group reviewed the precise position of the compact radio source Sagittarius A*, in the B1950.0 reference system (Gum \& Pawsey 1960) to be

$\alpha_{\mathrm{B} 1950.0}^{0}=17^{\mathrm{h}} 42^{\mathrm{m}} 37^{\mathrm{s}}, \delta_{\mathrm{B} 1950.0}^{0}=-28^{\circ} 57^{\prime}$,

which was afterward used to define the direction of the $x$-axis (GC) of the IAU1958 GalCS. According to the rigorous FK4-FK5 transformation proposed by Standish (1982) and Aoki et al. (1983), the position of the $\mathrm{Sgr} \mathrm{A}^{*}$ at J2000.0 is

$\alpha_{\mathrm{J} 2000.0}^{\mathrm{SgrA} *}=17^{\mathrm{h}} 45^{\mathrm{m}} 47^{\mathrm{s}} .7, \delta_{\mathrm{J} 2000.0}^{\mathrm{SgrA} *}=-28^{\circ} .58^{\prime} 09^{\prime \prime}$,

while the $x$ axis direction of the $\mathrm{J} 2000.0$ GalCS recommended by the HipParcos team (ESA 1997) is

$\alpha_{\mathrm{J} 2000.0}^{0}=17^{\mathrm{h}} 45^{\mathrm{m}} 37^{\mathrm{s}} .1991, \delta_{\mathrm{J} 2000.0}^{0}=-28^{\circ} 56^{\prime} 10^{\prime} .221$.

Using Very Long Baseline Array (VLBA) measurements, Reid \& Brunthaler (2004) derived the absolute position (referred to the ICRS) of the Sgr A* at the dynamical center of the Galaxy

$\alpha_{\text {Reid }}^{\text {SgrA }}=17^{\mathrm{h}} 45^{\mathrm{m}} 40^{\mathrm{s}} .0400, \delta_{\text {Reid }}^{\mathrm{SgrA} *}=-29^{\circ} 00^{\prime} 28^{\mathrm{s}} .138$.

Since the Milky Way is a gravitational system centered at the $\mathrm{GC}$, the stellar density should be highest in that region. We find from the 2MASS data set that the position of the peak density is

$\alpha_{2 \mathrm{MASS}}^{0}=17^{\mathrm{h}} 46^{\mathrm{m}} 6^{\mathrm{s}} .5328, \delta_{2 \mathrm{MASS}}^{0}=-28^{\circ} 54^{\prime} 16^{\prime \prime} .164$.

In the following section, we adopt the Sgr A* position of Reid $\&$ Brunthaler (2004) derived using direct radio observations as the direction of the GC because it is independent of complicated transformations.

\section{Constructing the Galactic coordinate system}

We denote the orthogonal GalCS using three principal axes $[x, y, z]$. The orientation of the IAU1958 and HIPPARCos (J2000.0) GalCSs are characterized by the coordinates of the NGP (z-axis) and the position angle of the GC ( $x$-axis). After this set of parameters has been determined, we can construct the GalCS in the corresponding equatorial reference system using the $3 \times 3$ matrix $\mathcal{N}$. In Sect. 3, we have derived several possible positions of the NGP (Galactic plane) $\left(\alpha^{\mathrm{p}}, \delta^{\mathrm{p}}\right)$ and the GC $\left(\alpha^{0}, \delta^{0}\right)$. However, these coordinates or angles cannot be used to construct the GalCS directly, because their directions in $\left(\alpha^{\mathrm{p}}, \delta^{\mathrm{p}}\right)$ and $\left(\alpha^{0}, \delta^{0}\right)$ were determined independently, either by catalog fitting or by observations, and they are not perpendicular to each other. In other words, a given GC may not lie on a given Galactic plane. We construct the GalCS in two different ways: (1) we assume that the $z$-axis coincides with the given direction of the NGP $\left(\alpha^{\mathrm{p}}, \delta^{\mathrm{p}}\right)$ then try to find the $x$ direction using the given GC coordinates $\left(\alpha^{0}, \delta^{0}\right) ;(2)$ we assume that the $x$-axis coincides with the given direction $\left(\alpha^{0}, \delta^{0}\right)$ of the GC then try to infer the orientation of the Galactic plane from catalog fitting. 


\subsection{Method 1: z-axis fixed condition}

We first assume that the $z$-axis of the GalCS points in the direction of the NGP $\left(\alpha^{z}=\alpha^{\mathrm{p}}, \delta^{z}=\delta^{\mathrm{p}}\right)$ that is fitted from either the 2MASS or the SPECFIND v2.0 catalog. Since the given GC does not lie on the Galactic plane ( $x-y$ plane), we try to find the nearest point $\left(\alpha^{x}, \delta^{x}\right)$ from the given GC $\left(\alpha^{0}, \delta^{0}\right)$ on the fixed Galactic plane $\left(\alpha^{\mathrm{p}}, \delta^{\mathrm{p}}\right)$. Evidently, $\left(\alpha^{x}, \delta^{x}\right)$ and $\left(\alpha^{0}, \delta^{0}\right)$ share a common position angle $\theta$ with respect to the north celestial pole as seen from the NGP, given by

$\tan \theta=\frac{\sin \left(\alpha^{0}-\alpha^{\mathrm{p}}\right)}{\cos \delta^{\mathrm{p}} \tan \delta^{0}-\sin \delta^{\mathrm{p}} \cos \left(\alpha^{0}-\alpha^{\mathrm{p}}\right)}$.

Substituting in both Eqs. (4) and (8), we find that

$\theta_{2 \text { MASS }}=123.044663150$.

On the basis of these values of $\alpha_{2 \mathrm{MASS}}^{\mathrm{p}}, \delta_{2 \mathrm{MASS}}^{\mathrm{p}}$, and $\theta_{2 \mathrm{MASS}}$, we derive our new equatorial-Galactic transformation matrix based on the 2MASS data set and the Sgr A* position (printed to 10 decimals, 1 milliarcsecond accuracy)

$$
\begin{aligned}
& \mathcal{N}_{2 \text { MASS }}= \\
& \left(\begin{array}{lll}
-0.0505347007 & -0.8719028362 & -0.4870643574 \\
+0.4897972973 & -0.4466482209 & +0.7487349160 \\
-0.8703705255 & -0.2007257110 & +0.4496268868
\end{array}\right) .
\end{aligned}
$$

If we adopt Eqs. (5) and (8) to compile the GalCS, the position angle of $x$-axis and the rotation matrix are

$\theta_{\text {SPECFIND }}=122.914360525$,

and

$$
\begin{aligned}
& \mathcal{N}_{\text {SPECFIND }}= \\
& \left(\begin{array}{lll}
-0.0518807421 & -0.8722226427 & -0.4863497200 \\
+0.4846922369 & -0.4477920852 & +0.7513692061 \\
-0.8731447899 & -0.1967483417 & +0.4459913295
\end{array}\right) .
\end{aligned}
$$

\section{2. $x$-axis fixed condition}

In the above calculations, the $z$-axis of a constructed GalCS coincides perfectly with the fitted NGP, but the $x$-axis direction does not match the given GC (Sgr A*), instead offset by several arcminutes. We now assume that the $x$-axis coincides with the given GC ( $\left.\alpha^{x}=\alpha_{0}, \delta^{x}=\delta_{0}\right)$, and determine the coordinates of the NGP ( $z$ direction) by fitting either the 2MASS or SPECFIND v2.0 catalogs with the same strategy as in Sect. 3. Since we have already determined two of the three parameters that define the GalCS, there remains only one parameter to be determined. We choose the position angle $\eta$ of the NGP (seen from the given GC) as the last unknown parameter. We refer to Fig. 5 for the definition of $\eta$. The J2000.0 reference value of $\eta$ is

$\eta_{\mathrm{J} 2000.0}=58.599$

and the equation for fitting is then

$\cos \delta^{0} \tan \delta=\sin \delta^{0} \cos \left(\alpha-\alpha^{0}\right)+\sin \left(\alpha-\alpha^{0}\right) \tan \eta$.

The equatorial coordinate system to GalCS transformation matrix using $\left(\alpha^{0}, \delta^{0}, \eta\right)$ has the form

$\mathcal{N}=\mathcal{R}_{1}(\eta) \mathcal{R}_{2}\left(-\delta^{0}\right) \mathcal{R}_{3}\left(\alpha^{0}\right)$

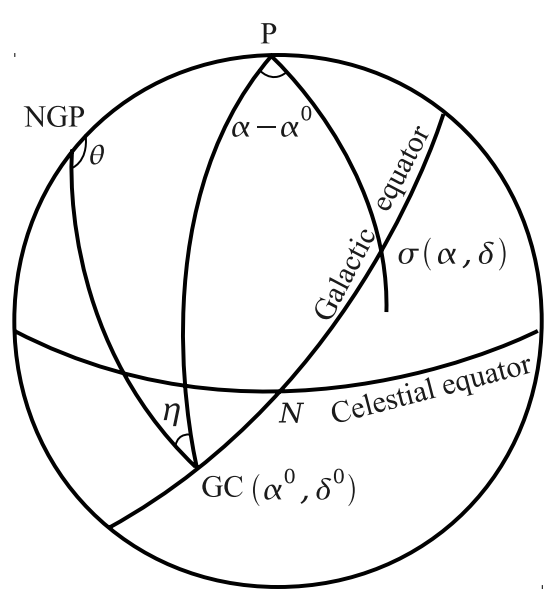

Fig. 5. The position angle $\eta$ of the NGP at the given $\mathrm{GC}\left(\alpha^{0}, \delta^{0}\right)$, where $\theta$ is the position angle of the $\mathrm{GC}, \sigma(\alpha, \delta)$ is a star on the Galactic equator, and $P$ is the celestial pole.

With the matrix $\mathcal{N}$, three standard parameters $\alpha^{\mathrm{p}}, \delta^{\mathrm{p}}$, and $\theta$ of the IAU scheme can be determined. In particular, we specify the coordinates in Eq. (8) to be the $x$-axis and fit the 2MASS stellar density data iteratively with Eq. (15) to determine $\eta$ and $\mathcal{N}$. The final results are

$\eta_{2 \mathrm{MASS}}=58^{\circ} .947 \pm 0.025$,

$$
\begin{aligned}
& \mathcal{N}_{2 \text { MASS }}= \\
& \left(\begin{array}{ll}
-0.0546572359 & -0.8728 \\
+0.4888603641 & -0.4468 \\
-0.8706481098 & -0.1961 \\
\alpha_{2 \text { MASS }}^{\mathrm{p}}=12^{\mathrm{h}} 50^{\mathrm{m}} 46^{\mathrm{s}} .5444, \\
\delta_{2 \text { MASS }}^{\mathrm{p}}=+26^{\circ} 48^{\prime} 56^{\prime \prime} .706, \\
\theta_{2 \text { MASS }}=122.912729244 .
\end{array}\right.
\end{aligned}
$$$$
\left(\begin{array}{lll}
-0.0546572359 & -0.8728439269 & -0.4849289286 \\
+0.4888603641 & -0.4468595864 & +0.7492209651 \\
-0.8706481098 & -0.1961121855 & +0.4511229097
\end{array}\right),
$$

Similarly, we obtain the orientation of the GalCS based on Eq. (8) and the SPECFIND v2.0 catalog

$\eta_{\text {SPECFIND }}=59^{\circ} .275 \pm 0.024$

$$
\begin{aligned}
& \mathcal{N}_{\text {SPECFIND }}= \\
& \left(\begin{array}{lll}
-0.0546572359 & -0.8728439269 & -0.4849289286 \\
+0.4838685275 & -0.4479748647 & +0.7517910405 \\
-0.8734322153 & -0.1935510264 & +0.4468267735
\end{array}\right), \\
& \alpha_{\text {SPECFIND }}^{\mathrm{p}}=12^{\mathrm{h}} 49^{\mathrm{m}} 58^{\mathrm{s}} .7360 \\
& \delta_{\text {SPECFIND }}^{\mathrm{p}}=+26^{\circ} 32^{\prime} 24^{\prime \prime} .989 \\
& \theta_{\text {SPECFIND }}=122^{\circ} .823292026 .
\end{aligned}
$$

The resulting values of $\eta$ from the both 2 MASS and the SPECFIND v2.0 differs by a few tenths of a degree when referred to $\eta_{\mathrm{J} 2000.0}$.

The GalCSs derived by applying the two methods with the same observational data (2MASS or SPECFIND) differ in terms of the small-angle orientation by several arcminutes, while the bias in the GalCSs caused by the use of different observational data is about a few tenths of an arcsec. In Sect. 4 of Liu et al. (2011), the authors proposed a possible definition of the GalCS based on the ICRS position of Sgr $\mathrm{A}^{*}$ and the transformed 
GalCS, while the present proposals depend only on the observational data but are unrelated to the old GalCS and its transformations between various fundamental reference systems, which should be avoided when defining a new coordinate system. In the context of our aim to establish a revised GalCS, we have succeeded in defining an optimal GalCS in which the $z$-axis coincides with the NGP and the $x$-axis with the position of Sgr $\mathrm{A}^{*}$, which is regarded as the GC. All data have been used were measured in the framework of the ICRS. The GalCS should be independent of epoch, thus the transformation matrix should be applied at the epoch of $\mathbf{J} 2000.0$. The results presented in Eqs. (8), (11), (13), (19), and (22) provide possible references for future revisions of the definition of the GalCS.

\section{Discussion and conclusion}

On the basis of near-infrared and radio observational data, we have described several approaches for calculating the positions of the Galactic plane and the GC. From our fitted results, we have found that the inclination of the Galactic plane with respect to the ICRS equator is either $0.4^{\circ}$ (2MASS) or $0.6^{\circ}$ (SPECFIND v2.0) larger than commonly used values recommended by the Hipparcos. This implies that there remains room for improvement in the J2000.0 GalCS.

As mentioned at the beginning of the paper, the main purpose of establishing a robust GalCS is to ensure that in studies of Galactic kinematics, dynamics, and structure, the Galactic coordinates $(\ell, b)$ of an object are always reliably and universally transformed from its equatorial coordinates $(\alpha, \delta)$. We note that the establishment of the GalCS need not have the same degree of precision as either the ecliptic or equatorial reference systems. The FK4, FK5, and Hipparcos or the ICRS are reference systems based on carefully determined positions of the equator and equinox, while the Galactic coordinate system is based on the concentration of tens of millions of stars or continuum radio objects in a statistical sense. We have defined in the previous sections a transformation method $\left(\alpha^{\mathrm{p}}, \delta^{\mathrm{p}}, \theta\right)$ that is the most appropriate choice for Galactic studies, at least at near-infrared and radio wavelengths. This transformation definition should be specified in certain fundamental reference systems, the ICRS. Our philosophy is similar to the IAU1958 method and the results are given for two bands, both based on the latest observations. The parameters presented in Sect. 4 provide some possible formal definitions of the GalCS that may be applied in the future.

With the development of astrometry to an unprecedented accuracy and the acquisition of a large amount of data in recent years, we recommend that the Galactic coordinate system be defined based on observational data rather than a transformation of the original definition. The optimal definition of GalCS remains an active field of research that should be considered by relevant IAU commissions.

Acknowledgements. This work was funded by the National Natural Science Foundation of China (NSFC) under No. 10973009 and made use of the SIMBAD database operated at CDS, Strasbourg, France. We are grateful to Dr. Yi Xie for his careful correction of the language.

\section{References}

Aoki, S., Soma, M., Kinoshita, H., \& Inoue, K. 1983, A\&A, 128, 263 Blaauw, A., Gum, C. S., Pawsey, J. L., \& Westerhout, G. 1960, MNRAS, 121, 123

Cutri, R. M., Sktutskie, M. F., van Dyk, S., et al. 2003, The IRSA 2MASS Allsky Point Source Catalog, NASA/IPAC Infrared Science Archive

ESA 1997, The Hipparcos and Tycho Catalogues (ESA SP-1200; Noordwijk: ESA)

Gilmore, G., \& Reid, N. 1983, MNRAS, 202, 1025

Gum, C. S., \& Pawsey, J. L. 1960, MNRAS, 121, 150

Liu, J.-C., Zhu, Z., \& Zhang, H. 2011, A\&A, 526, A16

Murray, C. A. 1989, A\&A, 218, 325

Miyamoto, M., Yoshizawa, M., \& Suzuki, S. 1988, A\&A, 194, 107

Monet, D. G., Levine, S. E., Casian, B., et al. 2003, AJ, 125, 984

Reid, M. J. 1993, ARA\&A, 31, 345

Reid, M. J., \& Brunthaler, A. 2004, ApJ, 616, 872

Roeser, S., Demleitner, M., \& Schilbach, E. 2010, AJ, 139, 2440

Skrutskie, M. F., Cutri, R. M., Stiening, R., et al. 2006, AJ, 131, 1163

Standish, E. M., Jr. 1982, A\&A, 115, 20

Veltz, L., Bienaymé, O., Freeman, K. C., et al. 2008, A\&A, 480, 753

Vollmer, B., Gassmann, B., Derriére, S., et al. 2010, A\&A, 511, A53

Westin, T. N. G. 1985, A\&AS, 60, 99

Zacharias, N., Finch, C., Girard, T., et al. 2010, AJ, 139, 2184

Zhu, Z. 2009, RAA, 9, 1285 